\title{
A NEW PROOF OF A THEOREM OF CASSELS AND PFISTER
}

\author{
LARRY J. GERSTEIN 1
}

\begin{abstract}
This note uses the theory of quadratic forms over Dedekind domains to give a new proof of a theorem of Cassels and Pfister on the representation of polynomials in terms of squares of rational functions.
\end{abstract}

The following theorem has been important in connection with studies of sums of squares.

THEOREM. Let $F$ be a field of characteristic not 2 , and let $f(x) \in F[x]$. Suppose there are scalars $\alpha_{1}, \cdots, \alpha_{n} \in F$ and rational functions $r_{1}(x), \cdots$, $r_{n}(x) \in F(x)$ such that

$$
f(x)=\alpha_{1}\left(r_{1}(x)\right)^{2}+\cdots+\alpha_{n}\left(r_{n}(x)\right)^{2} .
$$

Then there are polynomials $p_{1}(x), \cdots, p_{n}(x) \in F[x]$ such that

$$
f(x)=\alpha_{1}\left(p_{1}(x)\right)^{2}+\cdots+\alpha_{n}\left(p_{n}(x)\right)^{2} .
$$

This theorem evolved as follows. Artin proved [1, Satz 7] in 1927 that a polynomial $f(x) \in F[x]$ that is a sum of squares of rational functions in $F(x)$ can be expressed as a sum of squares of polynomials. In 1964 Cassels [2] showed that the same number of squares suffices. Then in 1965 Pfister [6] modified Cassels' proof to obtain the more general result stated above.

The proof to be given here uses the notions and terminology of quadratic spaces and lattices over Dedekind domains as in O'Meara [5]. Of particular interest are modular and maximal lattices over the polynomial ring $F[x]$. Since 2 is a unit of $F[x]$, every unimodular $F[x]$-lattice is $F[x]$-maximal, though the converse is false.

We need two lemmas. The proof of Lemma 1 follows the argument of Example 102:3 in [5]. Lemma 2 can be deduced by adapting Hermite's integral reduction (see [4, Theorem IV.17]) to the setting of quadratic

Presented to the Society, January 27, 1973 under the title Symmetric bilinear forms and a theorem of Cassels and Pfister; received by the editors April 30, 1973.

AMS (MOS) subject classifications (1970). Primary 10C05, 12E05; Secondary 13 F05.

1 This research was supported in part by the National Science Foundation under Grant GP-33044.

(c) American Mathematical Society 1973 
forms over $F[x]$. Alternately, it follows immediately from theorems of Harder [3, 13.4.3 and 13.4.8].

Lemma 1. Let $V$ be a regular quadratic $F(x)$-space, and let $L$ and $M$ be $F[x]$-maximal lattices on $V$. Then $L$ and $M$ have the same volume. In particular, if $V$ supports a unimodular lattice then every $F[x]$-maximal lattice on $V$ is unimodular.

Lemma 2. Let $L$ be a unimodular $F[x]$-lattice on an n-ary quadratic $F(x)$-space $V$. Then there are scalars $\alpha_{1}, \cdots, \alpha_{n} \in F$ such that $L \cong$ $\left\langle\alpha_{1}\right\rangle \perp \cdots \perp\left\langle\alpha_{n}\right\rangle$; and every unimodular $F[x]$-lattice on $V$ is isometric to $L$.

Proof of Theorem. Consider a quadratic $F(x)$-space $V$ given by $V \cong\left\langle\alpha_{1}\right\rangle \perp \cdots \perp\left\langle\alpha_{n}\right\rangle$ with respect to a base $\left\{v_{1}, \cdots, v_{n}\right\}$. By the hypothesis, there is a vector $v \in V$ such that $Q(v)=f(x)$, so there is an $F[x]$-maximal lattice $M$ on $V$ with $v \in M$. Note that the lattice $F[x] v_{1} \perp \cdots \perp F[x] v_{n}$ $\cong\left\langle\alpha_{1}\right\rangle \perp \cdots \perp\left\langle\alpha_{n}\right\rangle$ is unimodular. Hence, by Lemma $1, M$ is also unimodular, so by Lemma 2 there is an $F[x]$-base $\left\{w_{1}, \cdots, w_{n}\right\}$ for $M$ with respect to which $M \cong\left\langle\alpha_{1}\right\rangle \perp \cdots \perp\left\langle\alpha_{n}\right\rangle$. Now let $p_{1}(x), \cdots, p_{n}(x)$ be the coefficients of $v$ with respect to this basis, and the result follows.

\section{REFERENCES}

Q.E.D.

1. E. Artin, Über die Z.erlegung definiter Funktionen in Quadrate, Abh. Math. Sem. Hamburg 5 (1927), 100-115.

2. J. W. S. Cassels, On the representation of rational functions as sums of squares, Acta Arith. 9 (1964), 79-82. MR 29 \#95.

3. M. Knebusch, Grothendieck- und Wittringe von nichtausgearteten symmetrischen Bilinearformen, S.-B. Heidelberger Akad. Wiss. Math.-Natur. K1. 1969/70, 93-157. MR 42 \#6001.

4. M. Newman, Integral matrices, Academic Press, New York, 1972.

5. O. T. O'Meara, Introduction to quadratic forms, Die Grundlehren der math. Wissenschaften, Band 117, Academic Press, New York; Springer-Verlag, Berlin, 1963. MR 27 \#2485.

6. A. Pfister, Multiplikative quadratische Formen, Arch. Math. 16 (1965), 363-370. MR 32 \#2408.

Department of Mathematics, University of Notre Dame, Notre Dame, Indiana 46556

Current address: Department of Mathematics, University of California, Santa Barbara, California 93106 